Research Article

\title{
Optimal Pricing and Service Investment of Home-Sharing Platforms in a Duopoly Market
}

\author{
Yingna $\mathrm{Li}^{1}$ and Pengfei Ma ${ }^{2}{ }^{2}$ \\ ${ }^{1}$ Department of Management Engineering, Inner Mongolia Vocational and Technical College of Communications, \\ Chifeng 024005, Inner Mongolia, China \\ ${ }^{2}$ Business Management Department, CCTEG Shenyang Research Institute, Fushun 113112, Liaoning, China
}

Correspondence should be addressed to Pengfei Ma; mapengfei@syccri.com

Received 3 August 2021; Revised 4 November 2021; Accepted 15 November 2021; Published 24 December 2021

Academic Editor: Jun Li

Copyright (C) 2021 Yingna Li and Pengfei Ma. This is an open access article distributed under the Creative Commons Attribution License, which permits unrestricted use, distribution, and reproduction in any medium, provided the original work is properly cited.

\begin{abstract}
In this paper, we construct a fully covered duopoly market model. In this market, two home-sharing platforms provide differentiated rental services to consumers, respectively, and each platform has two strategies: short-term rental strategy and longterm rental strategy. This paper studies the pricing decisions and service investment of home-sharing platforms in a competitive market. The results show that, in the market equilibrium, how the platform chooses the strategy largely depends on the service quality of competitors. Specifically, when the difference in service quality is small, it is better for the two platforms to adopt the short-term rental strategy; otherwise, the two platforms are more inclined to adopt the long-term rental strategy. We also find that the commission rate and service cost will also affect the profitability of the platform. Finally, we extend the model to the uncovered market.
\end{abstract}

\section{Introduction}

In recent years, some online platforms have emerged in the accommodation industry to promote home-sharing, such as Airbnb, Homeway, Booking.com, and VRBO [1]. In these home-sharing platforms, service provides rent idle homes to consumers in need and obtain income [2]. This satisfies the needs of consumers and improves the overall utilization efficiency of the house $[3,4]$. The most popular homesharing platform is Airbnb. Airbnb is valued at over \$31 billion [5]. Airbnb has more than 6 million unique listings in more than 99800 cities in more than 191 countries and regions. As of May 2019, hosts on Airbnb have generated over $\$ 65$ billion in revenue [6].

The latest development in the field of home-sharing is several commercial operators to home-sharing platforms, such as Pillow.com, Marriott Bonvoy, and Tujia. These commercial operators collect the idle homes for unified operation and management and then rent them through the home-sharing platform. Different from the Consumer-to-
Consumer (C2C) home-sharing platform based on market pricing, these commercial operators have pricing power. They can set the prices on behalf of the hosts to maximize their profits and share the profits with the owners according to the predetermined proportion of income sharing [7].

For example, while most hosts on Airbnb are individuals, commercial operators are becoming increasingly influential [8]. In New York, 12 percent of Airbnb hosts are commercial operators, which account for 28 percent of total rental income, according to the data [9]. Meanwhile, more than 20 percent of listings are from hosts who own at least ten properties, indicating that the platform controlling all idle homes has become a phenomenon worthy of attention in the field of home-sharing.

The services provided by the platform often play a crucial role in influencing the utility of consumers [10]. For example, the platform can provide consumers with infrastructure, cleaning services, and a feedback mechanism for consumer evaluation. These services are important factors that influence consumers' purchasing decisions. These 
services play an important role in stimulating demand. In addition, the pricing decision of the platform is very important, which plays a decisive role in the profitability of the platform $[11,12]$. Therefore, we pay special attention to home-sharing platforms with pricing power.

Generally, each platform has two strategies to choose from short-term rental strategy and long-term rental strategy. Not all home-sharing platforms choose the shortterm rental strategy. If the platform chooses the short-term rental strategy, it will have more customers sources and wider geographical scope, but it also has the risk of a low occupancy rate. If the platform chooses the long-term leasing strategy, once the transaction is completed, it will have fixed consumers who come from the region. Although both the short-term rental strategy and the long-term rental strategy can be observed in the home-sharing market, it is difficult to judge which strategy should be chosen under what conditions when the platform controls all listed homes. Therefore, we focus on the following key issues:

(1) In the duopoly market, under what conditions will the platform adopt the short-term rental strategy better than the long-term rental strategy?

(2) How does the platform determine the optimal service price and service level?

(3) How do the commission rate and service cost affect the strategy selection of the platform?

The purpose of this paper is to provide reference and theoretical support for the operational decision-making and strategy selection of competitive platforms. Based on the practice of home-sharing platform, we consider that there are two competing platforms in the market and provide differentiated housing rental services, respectively. We analyze four cases: Case SS, both platforms choose short-term rental strategy; Case SL, platform 1 chooses the short-term rental strategy, and platform 2 chooses the long-term rental strategy; Case LS, platform 1 chooses the long-term rental strategy, and platform 2 chooses the short-term rental strategy; Case LL, both platforms choose the long-term rental strategy. Firstly, based on the Hotelling model, this paper constructs the profit maximization model of the platform in four different cases. Furthermore, it gives the optimal service price and service level in each case and analyzes the influence of service cost and commission rate on the optimal service price, service level, and profit. Finally, by comparing the platform's profits in different cases, we discuss the conditions under which the platforms adopt different strategies and under what conditions to achieve equilibrium.

The main contributions of this paper are as follows. Firstly, this paper focuses on the optimal pricing decisions and service investment of the home-sharing platform under the competitive environment and examines the impact of commission rate and service cost on the platform's profit. Existing studies have analyzed pricing models in the market through the demand response management framework of contract theory [13]. Our research can fill the gap of the existing research. Secondly, through theoretical analysis, we can gain some new conclusions. For example, under some conditions, there may be two equilibria; that is, under different circumstances, both platforms can get the optimal profits. Therefore, we also give the condition of dominant equilibrium.

The rest of this paper is as follows. Section 2 reviews the relevant literature. Section 3 gives the problem description. Section 4 gives the equilibrium price, service level, and profit of the two platforms under four cases. In Section 5, we analyze the market equilibrium and discuss the impact of the service cost and commission rate on the profits of the two platforms. In Section 6, we extend our analysis to markets that are not fully covered and give the equilibrium results of the two platforms. Section 7 summarizes the main conclusions and management implications of this paper and gives future research. All the proofs are shown in Appendix.

\section{Literature Review}

This study is related to two research streams: the business model and the pricing decisions of the home-sharing platform. Next, we review the existing research on these two streams and discuss the interaction between our research and the existing research.

With the application of Internet and mobile technology $[14,15]$, home-sharing platforms have developed rapidly. There is a large amount of literature that studies the business models of housing sharing platforms. For example, Zhu et al. point out that the current home-sharing platform has two business models: the business model priced by the market and the business model priced by the platform [7]. In the study of home-sharing based on market pricing, Zhou et al. find that the price is naturally determined by the supply and demand in the market; the purpose is to maintain the balance between supply and demand [16]. Moreover, Gibbs et al. point out that the price of listings on Airbnb is determined by the host based on the market price, and the platform only collects commissions and does not enforce pricing [17]. Zhu et al. point out that, compared with the home-sharing platform priced by the market, in the homesharing platform priced by the platform, although it is still owned by the individual owner voluntarily, the price is determined by the platform, and the goal of the platform is often to maximize its benefit [7]. Different from the above research, we focus on the optimal pricing decision and service level investment of home-sharing platforms under a competitive environment. We analyze different situations: both platforms adopt the short-term rental strategy, one platform adopts the short-term rental strategy, and the other adopts the long-term rental strategy; both platforms adopt the long-term rental strategy. Furthermore, the conditions of different strategies are further analyzed.

On the pricing decision of the home-sharing platform, we can see that scholars have carried out some research work. For example, Gal-Or studies the competition between the home-sharing platform and traditional hotels, and the results show that if the service fee of the platform is low enough, the consumer surplus will increase [18]. Gal-Or studies the impact of competition between home-sharing 
platforms on prices. They find that the competition can reduce the fees that platforms can charge consumers [19]. Zhou et al. study the competition between home-sharing platforms. They point out that, under certain conditions, two platforms will adopt the same commission rate contract [16]. $\mathrm{Li}$ et al. conduct an empirical study on the pricing behavior of experienced and inexperienced hosts on Airbnb. They find that experienced hosts are more likely to earn higher incomes because they often adjust prices and respond to high demand [20]. The platform can analyze consumers' preference for service quality by collecting data about transactions [21-23]. Hong and Yoo study the relationship between service price and pricing, and their findings deepen the understanding of hosts' pricing decisions [24]. Wang and Nicolau point out the factors that affect the rental price of Airbnb and also analyze the impact of the pricing of Airbnb on hotel channel performance [10]. Zervas et al. find that Airbnb hinders the development of low-end hotels and hotels that do not cater to business tourists. They also believe that, compared with hotels, the home-sharing platform has the advantages of low marginal costs and a wide range of services [25]. Barron et al. analyze the impact of homesharing on housing sales and rents through Airbnb's transaction data [26]. Different from the above research, we consider both the optimal pricing decision and service level investment for home-sharing platforms. We find that the service price and service level are related to factors such as the intrinsic value and commission rate of the services of its competitors.

\section{Problem Description}

In this paper, we consider that there are two home-sharing platforms (hereinafter referred to as "the platform") to collect individual hosts' homes, respectively, carry out unified decoration and management, and then for rental. Each platform has two strategies to choose from the shortterm rental strategy and the long-term rental strategy. Each consumer who chooses the short-term rental service can only rent one day at most, and each consumer who chooses the long-term rental service can only rent one period at most. The rental services of the two platforms are characterized by quality attributes (i.e., vertical differentiation) and suitability attributes (i.e., horizontal differentiation). All consumers prefer high-quality service, but different consumers have different preferences. Quality determines the intrinsic value obtained by consumers from products, and the suitability attribute describes the negative utility caused by the inappropriate relationship between consumers' ideal service and their expected service.

For the convenience of analysis, the definition and description of related symbols and variables involved in this paper are shown in Table 1 , where $i=\{1,2\}$ and $k \in\{\mathrm{SS}, \mathrm{SL}, \mathrm{LS}, \mathrm{LL}\}$.

\section{Model}

In this paper, we build the model based on the Hotelling model and assume that the market size is $N$. Each consumer only needs one service from two platforms at most, and the preferences of consumers are evenly divided in $[0,1]$, where platform 1 is located at 0 and platform 2 is located at 1 . A consumer's location represents his ideal service. If a service does not exactly match the consumer's preferences, then the consumer incurs inappropriate costs, which increase with the distance between his location and the given platform. Assume that the inappropriate cost for consumers to choose short-term rental service is $\lambda$. Since the rental time of longterm rental service is long, the inappropriate cost of longterm rental service is assumed to be $\beta \lambda$, where $\beta>1$. The utility functions of consumers are as follows:

$$
\begin{aligned}
& U_{S 1}=v_{S 1}-p_{1}^{k}+\alpha x_{1}^{k}-\lambda t, \\
& U_{L 1}=\frac{v_{L 1}-p_{1}^{k}}{N}+\alpha x_{1}^{k}-\beta \lambda t, \\
& U_{S 2}=v_{S 2}-p_{2}^{k}+\alpha x_{2}^{k}-\lambda(1-t), \\
& U_{L 2}=\frac{v_{L 2}-p_{2}^{k}}{N}+\alpha x_{2}^{k}-\beta \lambda(1-t) .
\end{aligned}
$$

Next, we will analyze the different strategies adopted by each platform.

4.1. Case SS: Both Platforms Choose the Short-Term Rental Strategy. When both platforms choose the short-term rental strategy, the utility of consumers choosing platforms 1 and 2 is equations (1) and (3), respectively, as shown in Figure 1.

The goal of each consumer is to maximize their utility. Therefore, the whole market is divided into $\left(0, t_{0}\right)$ and $\left(t_{0}, 1\right)$. Consumers in $\left(0, t_{0}\right)$ prefer to choose the service of platform 1 rather than that of platform 2. On the contrary, consumers in $\left(t_{0}, 1\right)$ prefer the service of platform 2 rather than that of platform 1 . The marginal consumers at $t_{0}$ have no difference in choosing the service of platform 1 and platform 2.

By $U_{S 1}=U_{S 2}$, we can obtain $t_{0}=\left(\left(\lambda-p_{1}^{S S}+p_{2}^{S S}+v_{S 1}-v_{S 2}+\alpha x_{1}^{S S}-\alpha x_{2}^{S S}\right) / 2 \lambda\right)$; then the demand functions of platform 1 and platform 2 are $D_{\mathrm{SS}}^{1}=t_{0}$ and $D_{S S}^{2}=1-t_{0}$, respectively.

Under Case SS, the profit functions of the platforms are, respectively, in equations (5) and (6):

$$
\begin{aligned}
& \Pi_{1}^{\mathrm{SS}}=r_{1} p_{1}^{\mathrm{SS}} D_{1}^{\mathrm{SS}} N-c\left(x_{1}^{\mathrm{SS}}\right)^{2}, \\
& \Pi_{2}^{\mathrm{SS}}=r_{2} p_{2}^{\mathrm{SS}} D_{2}^{\mathrm{SS}} N-c\left(x_{2}^{\mathrm{SS}}\right)^{2} .
\end{aligned}
$$

Each platform determines the optimal short-term rental service price $p_{i}^{S S^{*}}$ and the service level $x_{i}^{S S^{*}}$ to maximize its profit. The optimal solutions can be obtained by substituting $t_{0}$ into equations (5) and (6), respectively. In this paper, we assume that the second-order conditions are positive (i.e., $8 c \lambda-\alpha^{2} r_{1} N>0$ and $8 c \lambda-\alpha^{2} r_{2} N>0$ ), which can ensure that the demand functions of each platform are positive.

By solving the profit functions of platform 1 and platform 2, the following lemma can be obtained. 
TABLE 1: Summary of notations.

\begin{tabular}{lc}
\hline Notations & Descriptions \\
\hline$p_{i}^{k}$ & Service price (decision variable). \\
$x_{i}^{k}$ & Service level (decision variables). \\
$N$ & The number of days in a period. \\
$v_{S i}$ & The intrinsic value of short-term rental services. \\
$v_{L i}$ & The intrinsic value of long-term rental services. \\
$x_{i}^{k}$ & Utility per unit of service level. \\
$\lambda / \beta \lambda$ & The unsuitable cost for consumers to choose the short-term rental service/ the long-term rental service, where $\beta>1$. \\
$c$ & Unit cost of services provided by the platform. \\
$r_{i}$ & Commission rate. \\
\hline
\end{tabular}

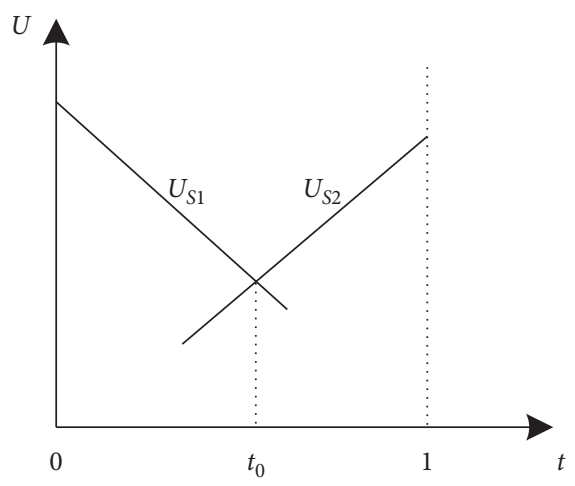

FIgURE 1: Market segmentation under Case SS.

Lemma 1. The optimal short-rental price and the service level of platform 1 and platform 2, are, respectively,

$$
\begin{aligned}
p_{1}^{S S^{*}} & =\frac{2 \lambda\left(6 c \lambda-\alpha^{2} r_{2} N+2 c v_{S 1}-2 c v_{S 2}\right)}{12 c \lambda-\alpha^{2} r_{1} N-\alpha^{2} r_{2} N}, \\
x_{1}^{S S^{*}} & =\frac{\alpha r_{1} N\left(6 c \lambda-\alpha^{2} r_{2} N+2 c v_{S 1}-2 c v_{S 2}\right)}{2 c\left(12 c \lambda-\alpha^{2} r_{1} N-\alpha^{2} r_{2} N\right)}, \\
p_{2}^{S S^{*}} & =\frac{2 \lambda\left(6 c \lambda-\alpha^{2} r_{1} N-2 c v_{S 1}+2 c v_{S 2}\right)}{12 c \lambda-\alpha^{2} r_{1} N-\alpha^{2} r_{2} N}, \\
x_{2}^{S S^{*}} & =\frac{\alpha r_{2} N\left(6 c \lambda-\alpha^{2} r_{1} N-2 c v_{S 1}+2 c v_{S 2}\right)}{2 c\left(12 c \lambda-\alpha^{2} r_{1} N-\alpha^{2} r_{2} N\right)} .
\end{aligned}
$$

Furthermore, the demand and profit of platform 1 can be obtained as

$$
\begin{aligned}
& D_{1}^{S S^{*}}=\frac{6 c \lambda-\alpha^{2} r_{2} N+2 c v_{S 1}-2 c v_{S 2}}{12 c \lambda-\alpha^{2} r_{1} N-\alpha^{2} r_{2} N}, \\
& \Pi_{1}^{S S^{*}}=\frac{r_{1} N\left(8 c \lambda-\alpha^{2} r_{1} N\right)\left(6 c \lambda-\alpha^{2} r_{2} N+2 c v_{S 1}-2 c v_{S 2}\right)^{2}}{4 c\left(12 c \lambda-\alpha^{2} r_{1} N-\alpha^{2} r_{2} N\right)^{2}} .
\end{aligned}
$$

The demand and the profit of platform 2 can be obtained as

$$
\begin{aligned}
& D_{2}^{S S^{*}}=\frac{6 c \lambda-\alpha^{2} r_{1} N-2 c v_{S 1}+2 c v_{S 2}}{12 c \lambda-\alpha^{2} r_{1} N-\alpha^{2} r_{2} N}, \\
& \Pi_{2}^{S S^{*}}=\frac{r_{2} N\left(8 c \lambda-\alpha^{2} r_{2} N\right)\left(6 c \lambda-\alpha^{2} r_{1} N-2 c v_{S 1}+2 c v_{S 2}\right)^{2}}{4 c\left(12 c \lambda-\alpha^{2} r_{1} N-\alpha^{2} r_{2} N\right)^{2}} .
\end{aligned}
$$

4.2. Case SL: Platform 1 Chooses the Short-Term Rental Strategy, and Platform 2 Chooses the Long-Term Rental Strategy. Under Case SL, we analyze the operational decisions of the two platforms. The utility obtained by consumers using the services of platform 1 and platform 2 is equations (1) and (4), respectively, as shown in Figure 2.

Similar to Case SS, the whole market is divided in $\left(0, t_{1}\right)$ and $\left(t_{1}, 1\right)$. The consumers in $\left(0, t_{1}\right)$ prefer to choose the service of platform 1 rather than that of platform 2 . On the contrary, the consumers in $\left(t_{1}, 1\right)$ prefer the service of platform 2 rather than that of platform 1. The marginal consumer at $t_{1}$ has no difference in choosing the service of platform 1 and platform 2 .

By $U_{S 1}=U_{L 2}$, we can obtain $t_{1}=\left(\left(N\left(v_{S 1}-\right.\right.\right.$ $\left.\left.\left.p_{1}^{\mathrm{SL}}+\beta \lambda+\alpha x_{1}^{\mathrm{SL}}-\alpha x_{2}^{\mathrm{SL}}\right)+p_{2}^{\mathrm{SL}}-v_{L 2}\right) / N \lambda(\beta+1)\right)$; then the demand functions of platform 1 and platform 2 are $D_{1}^{\mathrm{SL}}=t_{1}$ and $D_{\text {SS }}^{2}=1-t_{0}$, respectively.

Under Case SL, the profit functions of the platforms are, respectively, in equations (10) and (11): 


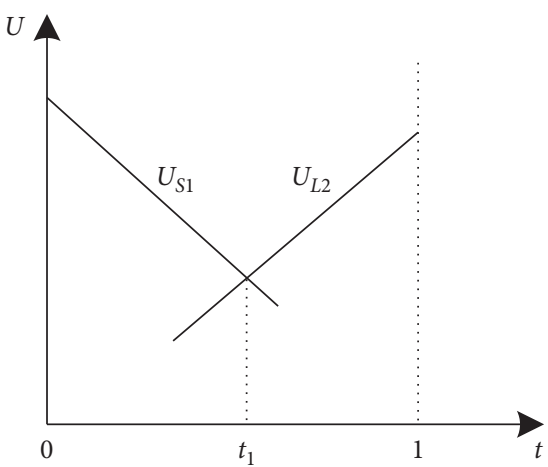

FIGURE 2: Market segmentation under Case SL.

$$
\begin{aligned}
& \Pi_{1}^{\mathrm{SL}}=r_{1} p_{1}^{\mathrm{SL}} D_{1}^{\mathrm{SL}} N-c\left(x_{1}^{\mathrm{SL}}\right)^{2}, \\
& \Pi_{2}^{\mathrm{SL}}=r_{2} p_{2}^{\mathrm{SL}} D_{2}^{\mathrm{SL}}-c\left(x_{2}^{\mathrm{SL}}\right)^{2} .
\end{aligned}
$$

Each platform determines the optimal short-term rental service price $p_{i}^{\mathrm{SL}}$ and the service level $x_{i}^{\mathrm{SL}}$ to maximize its profit. The optimal solutions can be obtained by substituting $t_{1}$ into equations (10) and (11), respectively. We assume that the second-order conditions are positive (i.e., $4 c \lambda+4 \beta c \lambda-$ $\alpha^{2} r_{1} N>0$ and $4 c \lambda+4 \beta c \lambda-\alpha^{2} r_{2} N>0$ ), which can ensure that the demand functions of each platform are positive.

By solving the profit functions of platform 1 and platform 2, the following lemma can be obtained.

Lemma 2. The optimal short-rental price and the service level of platform 1 and platform 2 are, respectively,

$$
\begin{aligned}
& p_{1}^{\mathrm{SL}}=\frac{\lambda(\beta+1)\left(2 c \lambda N+4 \beta c \lambda N-\alpha^{2} r_{2} N^{2}+2 c N v_{S 1}-2 c v_{L 2}\right)}{N\left(6 c \lambda+6 \beta c \lambda-\alpha^{2} r_{1} N-\alpha^{2} r_{2} N\right)}, \\
& x_{1}^{\mathrm{SL}}=\frac{\alpha r_{1}\left(2 c \lambda N+4 \beta c \lambda N-\alpha^{2} r_{2} N^{2}+2 c N v_{S 1}-2 c v_{L 2}\right)}{2 c\left(6 c \lambda+6 \beta c \lambda-\alpha^{2} r_{1} N-\alpha^{2} r_{2} N\right)}, \\
& p_{2}^{\mathrm{SL}}=\frac{\lambda(\beta+1)\left(2 \beta c \lambda N+4 c \lambda N-\alpha^{2} r_{1} N^{2}-2 c N v_{S 1}+2 c v_{L 2}\right)}{6 c \lambda+6 \beta c \lambda-\alpha^{2} r_{1} N-\alpha^{2} r_{2} N}, \\
& x_{2}^{\mathrm{SL}}=\frac{\alpha r_{2}\left(2 \beta c \lambda N+4 c \lambda N-\alpha^{2} r_{1} N^{2}-2 c N v_{S 1}+2 c v_{L 2}\right)}{2 c\left(6 c \lambda+6 \beta c \lambda-\alpha^{2} r_{1} N-\alpha^{2} r_{2} N\right)} .
\end{aligned}
$$

Furthermore, the demand and profit of platform 1 are as follows:

$$
\begin{aligned}
D_{1}^{S L^{*}} & =\frac{2 c \lambda N+4 \beta c \lambda N-\alpha^{2} r_{2} N^{2}+2 c N v_{S 1}-2 c v_{L 2}}{N\left(6 c \lambda+6 \beta c \lambda-\alpha^{2} r_{1} N-\alpha^{2} r_{2} N\right)}, \\
\Pi_{1}^{S L^{*}} & =\frac{r_{1}\left(4 c \lambda+4 \beta c \lambda-\alpha^{2} r_{1} N\right)\left(2 c \lambda N+4 \beta c \lambda N-\alpha^{2} r_{2} N^{2}+2 c N v_{S 1}-2 c v_{L 2}\right)^{2}}{4 c N\left(6 c \lambda+6 \beta c \lambda-\alpha^{2} r_{1} N-\alpha^{2} r_{2} N\right)^{2}} .
\end{aligned}
$$

The demand and profit of platform 2 are as follows:

$$
\begin{aligned}
D_{2}^{S L^{*}} & =\frac{2 \beta c \lambda N+4 c \lambda N-\alpha^{2} r_{1} N^{2}-2 c N v_{S 1}+2 c v_{L 2}}{N\left(6 c \lambda+6 \beta c \lambda-\alpha^{2} r_{1} N-\alpha^{2} r_{2} N\right)}, \\
\Pi_{2}^{S L^{*}} & =\frac{r_{2}\left(4 c \lambda+4 \beta c \lambda-\alpha^{2} r_{2} N\right)\left(2 \beta c \lambda N+4 c \lambda N-\alpha^{2} r_{1} N^{2}-2 c N v_{S 1}+2 c v_{L 2}\right)^{2}}{4 c N\left(6 c \lambda+6 \beta c \lambda-\alpha^{2} r_{1} N-\alpha^{2} r_{2} N\right)^{2}} .
\end{aligned}
$$

4.3. Case LS: Platform 1 Chooses the Long-Term Rental Strategy, and the Platform 2 Chooses the Short-Term Rental Strategy. The results of Case LS can be solved in the same way as Case SL. Under Case LS, the utility obtained by consumers using the services of platform 1 and platform 2 , respectively, is equations (2) and (3), as shown in Figure 3.

Similar to Case SL, by solving $U_{L 1}=U_{S 2}$, we can obtain

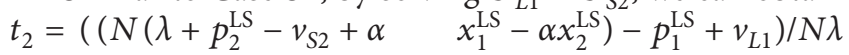
$(\beta+1))$; then the demand functions of platform 1 and platform 2 are $D_{1}^{\mathrm{LS}}=t_{2}$ and $D_{2}^{\mathrm{LS}}=1-t_{2}$.
Under Case LS, the profit functions of the platforms are, respectively, in equations (15) and (16):

$$
\begin{aligned}
& \Pi_{1}^{\mathrm{LS}}=r_{1} p_{1}^{\mathrm{LS}} D_{1}^{\mathrm{LS}}-c\left(x_{1}^{\mathrm{LS}}\right)^{2}, \\
& \Pi_{2}^{\mathrm{LS}}=r_{2} p_{2}^{\mathrm{LS}} D_{2}^{\mathrm{LS}} N-c\left(x_{2}^{\mathrm{LS}}\right)^{2} .
\end{aligned}
$$

Each platform determines the optimal service price $p_{i}^{\mathrm{LS}}$ and the service level $x_{i}^{\mathrm{LS}}$ to maximize its profit. The optimal solutions can be obtained by substituting $t_{2}$ into equations (15) and (16), respectively. We assume that the second-order 


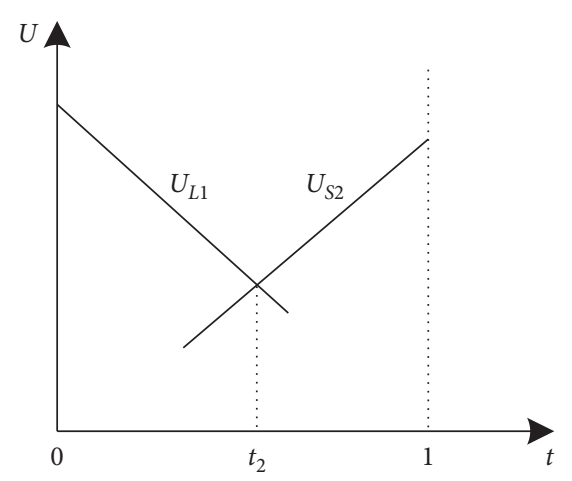

FIGURE 3: Market segmentation under Case LS.

conditions are positive (i.e., $4 c \lambda+4 \beta c \lambda-\alpha^{2} r_{1} N>0$ and $4 c \lambda+4 \beta c \lambda-\alpha^{2} r_{2} N>0$ ), which can ensure that the demand functions of each platform are positive.

By solving the profit functions of platform 1 and platform 2 , the following lemma can be obtained.

Lemma 3. The optimal short-rental price and the service level of platform 1 and platform 2 are, respectively,

$$
\begin{aligned}
& p_{1}^{\mathrm{LS} S^{*}}=\frac{\lambda(\beta+1)\left(2 \beta c \lambda N+4 c \lambda N-\alpha^{2} r_{2} N^{2}+2 c v_{L 1}-2 c N v_{S 2}\right)}{6 c \lambda+6 \beta c \lambda-\alpha^{2} r_{1} N-\alpha^{2} r_{2} N}, \\
& x_{1}^{\mathrm{LS} S^{*}}=\frac{\alpha r_{1}\left(2 \beta c \lambda N+4 c \lambda N-\alpha^{2} r_{2} N^{2}+2 c v_{L 1}-2 c N v_{S 2}\right)}{2 c\left(6 c \lambda+6 \beta c \lambda-\alpha^{2} r_{1} N-\alpha^{2} r_{2} N\right)}, \\
& p_{2}^{\mathrm{LS} S^{*}}=\frac{\lambda(\beta+1)\left(2 c \lambda N+4 \beta c \lambda N-\alpha^{2} r_{1} N^{2}-2 c v_{L 1}+2 c N v_{S 2}\right)}{N\left(6 c \lambda+6 \beta c \lambda-\alpha^{2} r_{1} N-\alpha^{2} r_{2} N\right)}, \\
& x_{2}^{\mathrm{LS}}=\frac{\alpha r_{2}\left(2 c \lambda N+4 \beta c \lambda N-\alpha^{2} r_{1} N^{2}-2 c v_{L 1}+2 c N v_{S 2}\right)}{2 c\left(6 c \lambda+6 \beta c \lambda-\alpha^{2} r_{1} N-\alpha^{2} r_{2} N\right)} .
\end{aligned}
$$

$$
\begin{aligned}
D_{1}^{\mathrm{LS}} & =\frac{2 \beta c \lambda N+4 c \lambda N-\alpha^{2} r_{2} N^{2}+2 c v_{L 1}-2 c N v_{S 2}}{N\left(6 c \lambda+6 \beta c \lambda-\alpha^{2} r_{1} N-\alpha^{2} r_{2} N\right)}, \\
\Pi_{1}^{\mathrm{LS}} & =\frac{r_{1}\left(4 c \lambda+4 \beta c \lambda-\alpha^{2} r_{1} N\right)\left(2 \beta c \lambda N+4 c \lambda N-\alpha^{2} r_{2} N^{2}+2 c v_{L 1}-2 c N v_{S 2}\right)^{2}}{4 c N\left(6 c \lambda+6 \beta c \lambda-\alpha^{2} r_{1} N-\alpha^{2} r_{2} N\right)^{2}} .
\end{aligned}
$$

The demand and profit of platform 2 are as follows:

$$
\begin{aligned}
& D_{2}^{\mathrm{LS}}=\frac{2 c \lambda N+4 \beta c \lambda N-\alpha^{2} r_{1} N^{2}-2 c v_{L 1}+2 c N v_{S 2}}{N\left(6 c \lambda+6 \beta c \lambda-\alpha^{2} r_{1} N-\alpha^{2} r_{2} N\right)}, \\
& \Pi_{2}^{\mathrm{LS}}=\frac{r_{2}\left(4 c \lambda+4 \beta c \lambda-\alpha^{2} r_{2} N\right)\left(2 c \lambda N+4 \beta c \lambda N-\alpha^{2} r_{1} N^{2}-2 c v_{L 1}+2 c N v_{S 2}\right)^{2}}{4 c N\left(6 c \lambda+6 \beta c \lambda-\alpha^{2} r_{1} N-\alpha^{2} r_{2} N\right)^{2}} .
\end{aligned}
$$


4.4. Case LL: Both Platforms Choose the Long-Term Rental Strategy. Under Case LL, the utility obtained by the consumers using the services of platform 1 and platform 2, respectively, is equations (2) and (4), as shown in Figure 4.

Similar to Case LS, by solving $U_{L 1}=U_{L 2}$, we can obtain $t_{3}=\left(\left(N\left(\beta \lambda+\alpha x_{1}^{\mathrm{LL}}-\alpha x_{2}^{L L}\right)-p_{1}^{\mathrm{LL}}+p_{2}^{\mathrm{LL}}+v_{L 1}-v_{L 2}\right) / 2 \beta \lambda\right.$ $N)$; then the demand functions of platform 1 and platform 2 are $D_{1}^{\mathrm{LL}}=t_{3}$ and $D_{2}^{\mathrm{LL}}=1-t_{3}$.

Under Case LL, the profit functions of the platforms are, respectively, in equations (20) and (21):

$$
\begin{aligned}
& \Pi_{1}^{\mathrm{LL}}=r_{1} p_{1}^{\mathrm{LL}} D_{1}^{\mathrm{LL}}-c\left(x_{1}^{\mathrm{LL}}\right)^{2}, \\
& \Pi_{2}^{\mathrm{LL}}=r_{2} p_{2}^{\mathrm{LL}} D_{2}^{\mathrm{LL}}-c\left(x_{2}^{\mathrm{LL}}\right)^{2} .
\end{aligned}
$$

Each platform determines the optimal service price $p_{i}^{\mathrm{LL}}$ and the service level $x_{i}^{\mathrm{LL}}$ to maximize its profit. The optimal solutions can be obtained by substituting $t_{3}$ into equations (20) and (21), respectively. We assume that the second-order conditions are positive (i.e., $8 \beta c \lambda-\alpha^{2} r_{1} N>0$ and $8 \beta c \lambda-\alpha^{2} r_{1} N>0$ ), which can ensure that the demand functions of each platform are positive.

By solving the profit functions of platform 1 and platform 2, the following lemma can be obtained.

Lemma 4. The optimal short-rental price and the service level of platform 1 and platform 2 are, respectively,

$$
\begin{aligned}
& p_{1}^{\mathrm{LL}}=\frac{2 \beta \lambda\left(6 \beta c \lambda N-\alpha^{2} r_{2} N+2 c v_{L 1}-2 c v_{L 2}\right)}{12 \beta c \lambda-\alpha^{2} r_{1} N-\alpha^{2} r_{2} N}, \\
& x_{1}^{\mathrm{LL}}=\frac{\alpha r_{1}\left(6 \beta c \lambda N-\alpha^{2} r_{2} N^{2}+2 c v_{L 1}-2 c v_{L 2}\right)}{2 c\left(12 \beta c \lambda-\alpha^{2} r_{1} N-\alpha^{2} r_{2} N\right)}, \\
& p_{2}^{\mathrm{LL}}=\frac{2 \beta \lambda\left(6 \beta c \lambda N-\alpha^{2} r_{1} N^{2}-2 c v_{L 1}+2 c v_{L 2}\right)}{12 \beta c \lambda-\alpha^{2} r_{1} N-\alpha^{2} r_{2} N}, \\
& x_{2}^{\mathrm{LL}}=\frac{\alpha r_{2}\left(6 \beta c \lambda N-\alpha^{2} r_{1} N^{2}-2 c v_{L 1}+2 c v_{L 2}\right)}{2 c\left(12 \beta c \lambda-\alpha^{2} r_{1} N-\alpha^{2} r_{2} N\right)} .
\end{aligned}
$$
follows:

$$
\begin{aligned}
D_{1}^{\mathrm{LL}} & =\frac{6 \beta c \lambda N-\alpha^{2} r_{2} N+2 c v_{L 1}-2 c v_{L 2}}{N\left(12 \beta c \lambda-\alpha^{2} r_{1} N-\alpha^{2} r_{2} N\right)}, \\
\Pi_{1}^{\mathrm{LL}} & =\frac{r_{1}\left(8 \beta c \lambda-\alpha^{2} r_{1} N\right)\left(6 \beta c \lambda N-\alpha^{2} r_{2} N^{2}+2 c v_{L 1}-2 c v_{L 2}\right)^{2}}{4 c N\left(12 \beta c \lambda-\alpha^{2} r_{1} N-\alpha^{2} r_{2} N\right)^{2}} .
\end{aligned}
$$

The demand and profit of platform 2 are as follows:

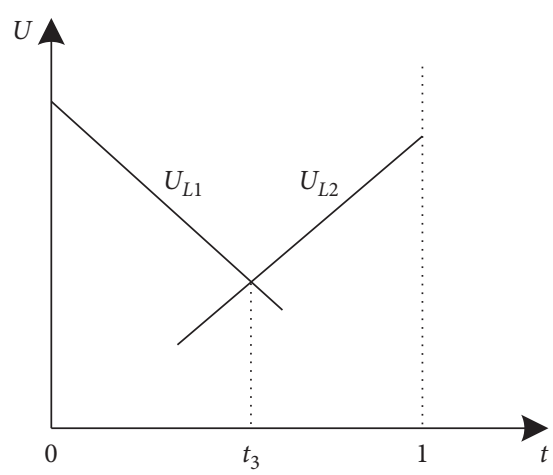

FIgURE 4: Market segmentation under Case LL.

$$
\begin{aligned}
D_{2}^{\mathrm{LL}} & =\frac{6 \beta c \lambda N-\alpha^{2} r_{1} N^{2}-2 c v_{L 1}+2 c v_{L 2}}{N\left(12 \beta c \lambda-\alpha^{2} r_{1} N-\alpha^{2} r_{2} N\right)}, \\
\Pi_{2}^{\mathrm{LL}} & =\frac{r_{2}\left(8 \beta c \lambda-\alpha^{2} r_{2} N\right)\left(6 \beta c \lambda N-\alpha^{2} r_{1} N^{2}-2 c v_{L 1}+2 c v_{L 2}\right)^{2}}{4 c N\left(12 \beta c \lambda-\alpha^{2} r_{1} N-\alpha^{2} r_{2} N\right)^{2}} .
\end{aligned}
$$

\section{Market Equilibrium}

The game is divided into three stages. First, platform 1 and platform 2 simultaneously decide whether to adopt the short-term or long-term rental strategy. Next, the two platforms determine the service price and the service level at the same time. Finally, consumers make purchase decisions after obtaining market information (e.g., service price, the rental strategy of the platform). In addition, due to the complexity of the model of duopoly market, we also give some numerical analysis to visually display the results to obtain more management insights, in which the parameter is set to $v_{L 1}=309, v_{L 2}=306, v_{s 1}=10, v_{s 2}=9.8, \beta=1.2$, $\lambda=13, \alpha=7, N=30, r_{1}=0.56$, and $r_{2}=0.55$.

In this paper, the equilibrium results under four different cases can be obtained by backward induction. In each subgame, we can obtain the optimal service price, service level, and profit of the two platforms. Table 2 summarizes the profits of the two platforms. By solving the payoff matrix in Table 2, the following proposition is obtained.

Proposition 1. The market equilibria under different conditions are as follows:

(i) if $\Delta V_{1}<T_{1}$, and $\Delta V_{2}<T_{2}$, Case $S S$ is an equilibrium;

(ii) if $\Delta V_{1}<T_{3}$, and $\Delta V_{2}>T_{2}$, Case $S L$ is an equilibrium;

(iii) if $\Delta V_{1}>T_{1}$, and $\Delta V_{2}<T_{4}$, Case LS is an equilibrium;

(iv) if $\Delta V_{1}>T_{3}$, and $\Delta V_{2}>T_{4}$, Case $L L$ is an equilibrium;

where $\Delta V=v_{S 1}-v_{S 2}, \Delta V_{1}=v_{L 1}-N v_{S 1} \Delta V_{2}=v_{L 2}-N v_{S 2}$, 
TABLE 2: Equilibrium profits.

Platform 2

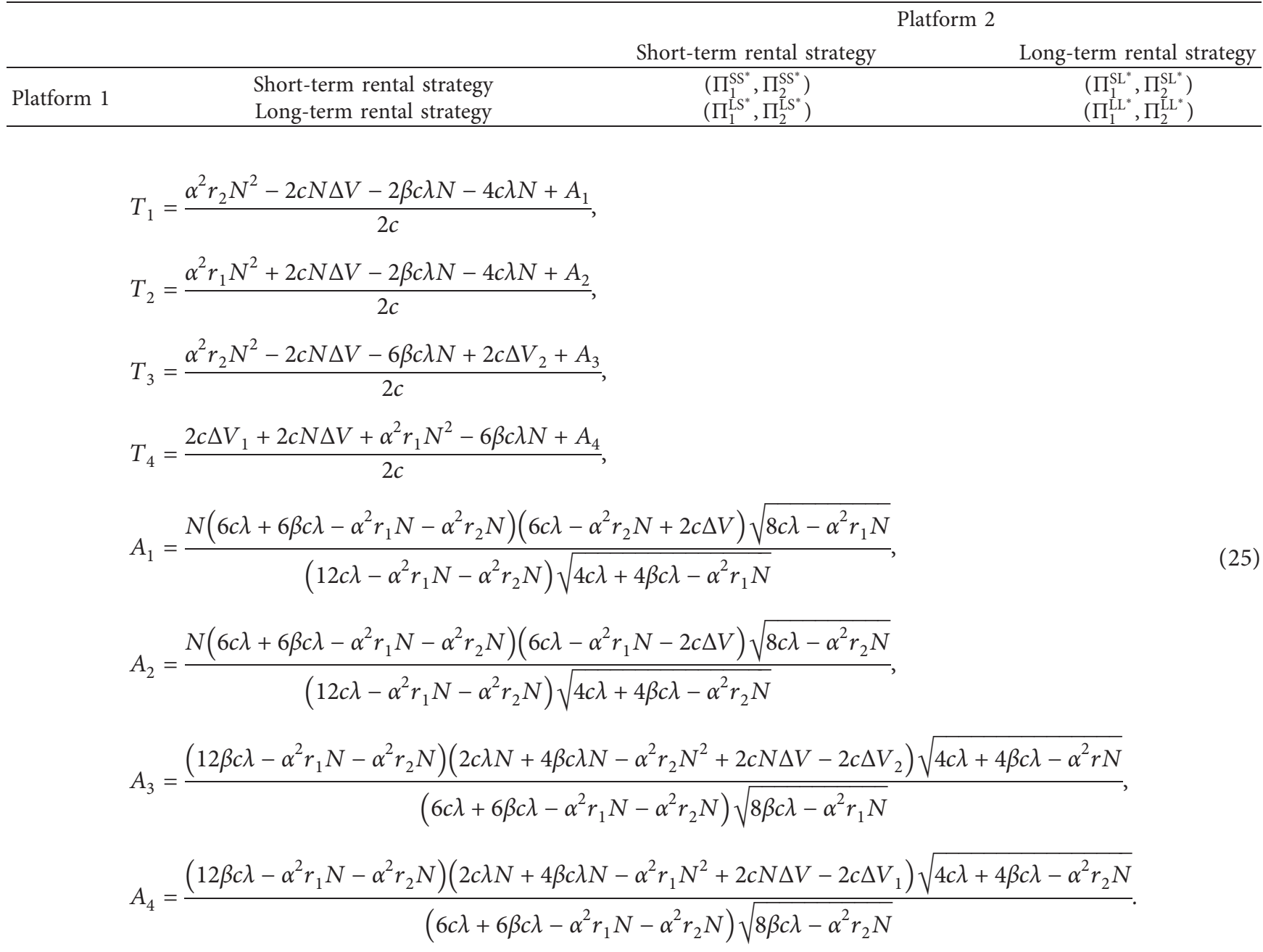

Proposition 1 describes four possible conditions of market equilibrium. Specifically, if the difference in the intrinsic values of the service $\left(\Delta V_{1}\right.$ and $\left.\Delta V_{2}\right)$ is small, then both platforms choose to adopt the short-term rental strategy in equilibrium. If the difference in the intrinsic values of the service (i.e., $\Delta V_{1}$ and $\left.\Delta V_{2}\right)$ is high enough, then the two platforms adopt the longterm rental strategy. When the intrinsic value of the service of platform 1 is high and and the intrinsic value of the service of platform 2 is small, then the platform 1 adopts the short-term rental strategy, while platform 2 adopts the long-term rental strategy.

When choosing the rental strategy, the platform must consider the intrinsic value of the services provided by competitors. If there is a significant difference in the intrinsic value difference between the services of the two platforms, the platform with the high intrinsic value difference chooses to adopt a long-term rental strategy in equilibrium, while the competitor chooses to adopt the short-term rental strategy. When faced with competitors with higher quality services, services can attract more consumers and expand the consumer base. Therefore, it is more profitable to choose the long-term rental strategy for a platform with high service quality. If the difference in the intrinsic value of services of each platform is significant, there are two situations: if the difference in the intrinsic value of services is small, the two platforms adopt the short-term rental strategy when in equilibrium; if there is a large difference in the intrinsic value of services, the two platforms will adopt the long-term rental strategy when they are in equilibrium.

However, when the quality difference between the longterm rental service and the short-term rental service is moderate, there are two equilibria in both cases, as shown in Proposition 2.

Proposition 2. Table 3 summarizes the conditions under which the two equilibria exist and the corresponding dominant equilibria, as shown in Figure 5.

In Table 3, we can find that when the threshold of the difference in service quality satisfies $T_{1}<T_{3}$ and $T_{2}<T_{4}$, there may be two equilibria in some similar regions. As shown in Figure 5(a), the region A-B-C-D is marked as SL/LS. We can also find that when the threshold of the difference in service quality satisfies $T_{3}<T_{1}$ and $T_{4}<T_{2}$, there are two equilibria in some similar regions; that is, both platforms adopt the 
TABLE 3: Two equilibria.

\begin{tabular}{lcc}
\hline Two equilibria & Dominant equilibrium & Parameter condition \\
\hline \multirow{2}{*}{ SS and LL } & \multirow{2}{*}{ SS } & $T_{3}<\Delta V_{1}<T_{1}$ and \\
& & $T_{4}<\Delta V_{2}<T_{2}$ \\
SL and LS & - & $T_{1}<\Delta V_{1}<T_{3}$ and \\
& & $T_{2}<\Delta V_{2}<T_{4}$ \\
\hline
\end{tabular}

short-term rental strategy or the long-term rental strategy. As shown in Figure 5(b), the region E-F-G-H is marked as SS/LL. This is because a platform's competitors choose the short-term rental strategy; there are two reasons for the platform to give up the long-term rental strategy. One is the quality of its service, and the other is to compete with it for more consumers. The more the competitor's customer base, the greater the consumer's demand for rental services. Therefore, the platform will lose market share and exit the market.

\section{Proposition 3}

(i) If both platforms choose the short-term rental strategy in equilibrium, when $r_{i}<\left(4 c \lambda\left(12 c \lambda-\alpha^{2}\right.\right.$ $\left.\left.r_{j} N\right) / \alpha^{2} N\left(8 c \lambda-\alpha^{2} r_{j} N\right)\right), \quad\left(\partial \Pi_{i} / \partial r_{i}\right)>0, \quad$ and $\left(\partial \Pi_{i} / \partial r_{j}\right)>0$.

(ii) If the platform $i$ chooses the short-term rental strategy and the platform $j$ chooses the long-term rental strategy in equilibrium, when $r_{i}<(2 c \lambda(1+\beta)[6 c \lambda$ $\left.\left.(1+\beta)-\alpha^{2} r_{j} N\right] / \alpha^{2} N\left(4 c \lambda+4 \beta c \lambda-\alpha^{2} r_{j} N\right)\right),\left(\partial \Pi_{i} /\right.$ $\left.\partial r_{i}\right)>0$, and $\left(\partial \Pi_{i} / \partial r_{j}\right)>0$.

(iii) If both platforms choose the log-term rental strategy in equilibrium, when $r_{i}>\left(4 \beta c \lambda\left(12 \beta c \lambda-\alpha^{2} r_{j} N\right) / \alpha^{2}\right.$ $\left.N\left(8 \beta c \lambda-\alpha^{2} r_{j} N\right)\right), \quad\left(\partial \Pi_{i} / \partial r_{i}\right)>0$, and $\left(\partial \Pi_{i} / \partial r_{j}\right)$ $>0$, where $i, j \in\{1,2\}, i \neq j$.

Proposition 3 shows that when both platforms choose the short-term rental strategy or the long-term rental strategy, the profit of platform $i$ increases with the increase of its commission rate $\left(r_{i}\right)$; no matter how $r_{i}$ changes, the profit of platform $i$ increases with the increase of commission rate $\left(r_{j}\right)$ of the competitive platform. In other words, if any commission rate increases, the profit of the platform will increase. The difference is that when the two platforms choose different rental strategies, the profit of platform $i$ increases with the increase of its commission rate $\left(r_{i}\right)$; no matter how $r_{i}$ changes, the profit of platform $i$ decreases with the increase of commission rate $\left(r_{j}\right)$ of competitive platform.

Proposition 4. In equilibrium, there are $\left(\partial \Pi_{i} / \partial c\right)>0$ and $\left(\partial \prod_{j} / \partial c\right)>0$, where $i, j \in\{1,2\}, i \neq j$.

Proposition 4 shows that regardless of whether platform $i$ and platform $j$ choose the short-term rental strategy or the long-term rental strategy, the profits of platform $i$ and platform $j$ increase with their service cost $c$ increases. The platform can reduce service costs and make it more profitable under the premise of ensuring service quality.

From Figures 6-9, we can find that the results of numerical analysis are consistent with those of Proposition 4. It can be seen from Figures 6-8 that, in the equilibrium state, the profit of platform 2 is larger than that of platform 1 under Cases SS, SL, and LS. In Figure 9, the profit of platform 1 is larger than that of platform 2 under Case LL. Furthermore, the platform can actually estimate the service cost of the industry through sufficient market research.

\section{Extension}

In the previous sections, this paper assumes that the market is fully covered. In this section, we relax this assumption to explore the market strategy and operational decisions of home-sharing platforms as local monopoly platforms.

6.1. Case SS: Both Platforms Are Local Monopolies and Both Platforms Choose the Short-Term Rental Strategy. When the market is not fully covered, both platforms are local monopolies. The marginal consumers who choose the service of platform 1 are at $t_{1 e}=\left(\left(v_{S 1}-p_{1 e}^{S S}+\alpha x_{1 e}^{S S}\right) / \lambda\right)$, and the marginal consumers who choose the service of platform 2 are at $t_{2 e}=\left(\left(\lambda+p_{2 e}^{\mathrm{SS}}-v_{S 2}-\alpha x_{2 e}^{\mathrm{SS}}\right) / \lambda\right)$. The consumers between $\left[0, t_{1 e}\right]$ choose the short-term rental service of platform 1 , and the consumers between $\left[t_{2 e}, 1\right]$ choose the shortterm rental service of platform 2 , where $t_{1 e}<t_{2 e}$. In this setting, these platforms do not directly compete with marginal consumers. Therefore, the profit functions of platform 1 and platform 2 are as follows: $\Pi_{1 e}^{\mathrm{SS}}=r_{1} p_{1 e}^{\mathrm{SS}} t_{1 e} N-$ $c\left(x_{1 e}^{\mathrm{SS}}\right)^{2}$ and $\Pi_{2 e}^{\mathrm{SS}}=r_{2} p_{2 e}^{\mathrm{SS}}\left(1-t_{2 e}\right) N-c\left(x_{2 e}^{\mathrm{SS}}\right)^{2}$. In equilibrium, the platforms determine the prices and the service levels, respectively, to maximize the profits. According to the first-order conditions of the profit functions on price and service level, the price and service level of platform 1 under equilibrium can be obtained as $p_{1 e}^{\mathrm{SS}}=\left(2 c \lambda v_{S 1}\right.$ $\left./\left(4 c \lambda-\alpha^{2} r_{1} N\right)\right), \quad p_{1 e}^{S S^{*}}=\left(2 c \lambda v_{S 1} /\left(4 c \lambda-\alpha^{2} r_{1} N\right)\right), \quad$ and $x_{1 e}^{S^{*}}=\left(\alpha r_{1} v_{S 1} N /\left(4 c \lambda-\alpha^{2} r_{1} N\right)\right)$. Furthermore, the demand and profit of platform 1 can be obtained as $D_{1 e}^{S S^{*}}=\left(2 c v_{S 1} /\left(4 c \lambda-\alpha^{2} r_{1} N\right)\right)$ and $\Pi_{1 e}^{S S^{*}}=\left(c r_{1} v_{S 1}^{2} N /(4 c \lambda-\right.$ $\left.\alpha^{2} r_{1} N\right)$ ). Similarly, we can get that the price and the service level of platform 2 are $p_{2 e}^{S S^{*}}=\left(2 c \lambda v_{S 2} /\left(4 c \lambda-\alpha^{2} r_{2} N\right)\right)$ and $x_{2 e}^{S S^{*}}=\left(\alpha r_{2} v_{S 2} N /\left(4 c \lambda-\alpha^{2} r_{2} N\right)\right)$. Furthermore, the demand and profit of platform 2 are $D_{2 e}^{S S^{*}}=\left(2 c v_{S 2} /\left(4 c \lambda-\alpha^{2} r_{2} N\right)\right)$ and $\Pi_{2 e}^{S S^{*}}=\left(c r_{2} v_{S 2}^{2} N /\left(4 c \lambda-\alpha^{2} r_{2} N\right)\right)$.

We need to ensure that $0<t_{1 e}<t_{2 e}<1$, so that both platforms can act as local monopolies. To ensure $t_{1 e}<t_{2 e}, 2 c$ $\left[v_{S 1}\left(4 c \lambda-\alpha^{2} r_{2} N\right)+v_{S 2}\left(4 c \lambda-\alpha^{2} r_{1} N\right)\right]<\left(4 c \lambda-\alpha^{2} r_{1} N\right)(4 c$ $\left.\lambda-\alpha^{2} r_{2} N\right)$ must be met. If this condition is not met, the two platforms will directly complete with marginal consumers.

6.2. Case SL: Both Platforms Are Local Monopolies, Platform 1 Chooses the Short-Term Rental Strategy, and Platform 2 Chooses the Long-Term Rental Strategy. When platform 1 chooses the short-term rental strategy and platform 2 chooses the long-term rental strategy, the marginal consumers who choose the service of platform 1 are at $t_{1 e}=\left(\left(v_{S 1}-p_{1 e}^{\mathrm{SL}}+\alpha x_{1 e}^{\mathrm{SL}}\right) / \lambda\right)$, and the marginal consumers who choose the service of platform 2 are at $t_{2 e}=\left(\left(\beta \lambda N-\alpha x_{2 e}^{\mathrm{SL}} N+p_{2 e}^{\mathrm{SL}}-v_{L 2}\right) / \beta \lambda N\right)$. The consumers 


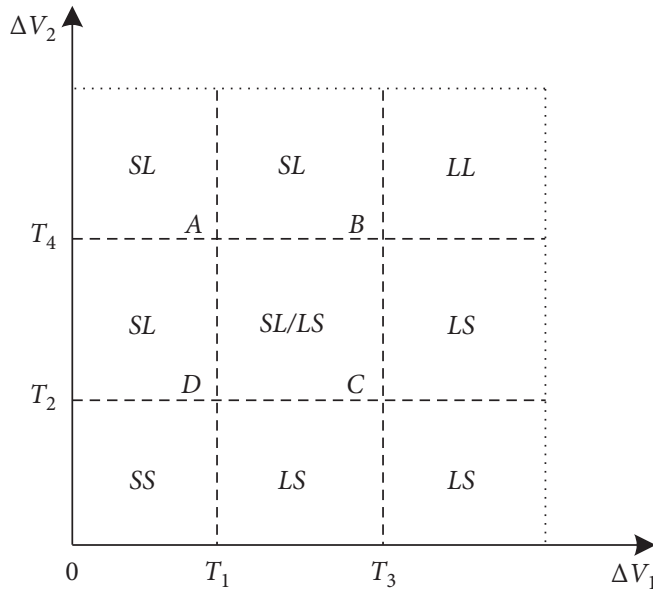

(a)

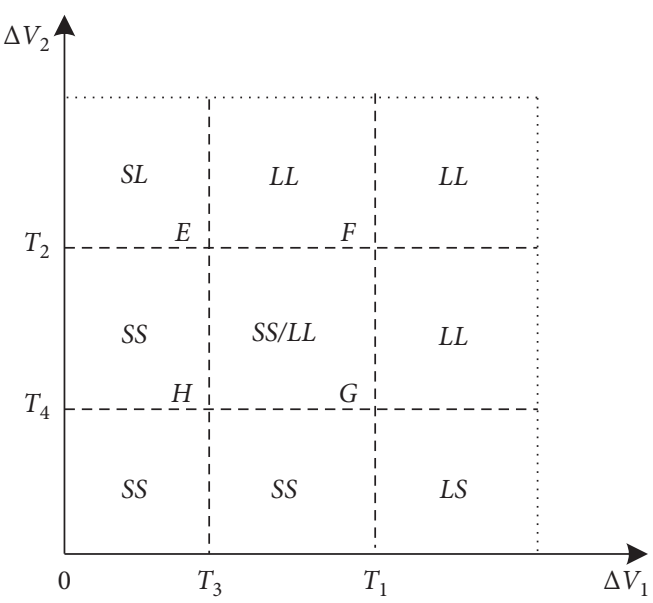

(b)

FIgURe 5: Two equilibria.

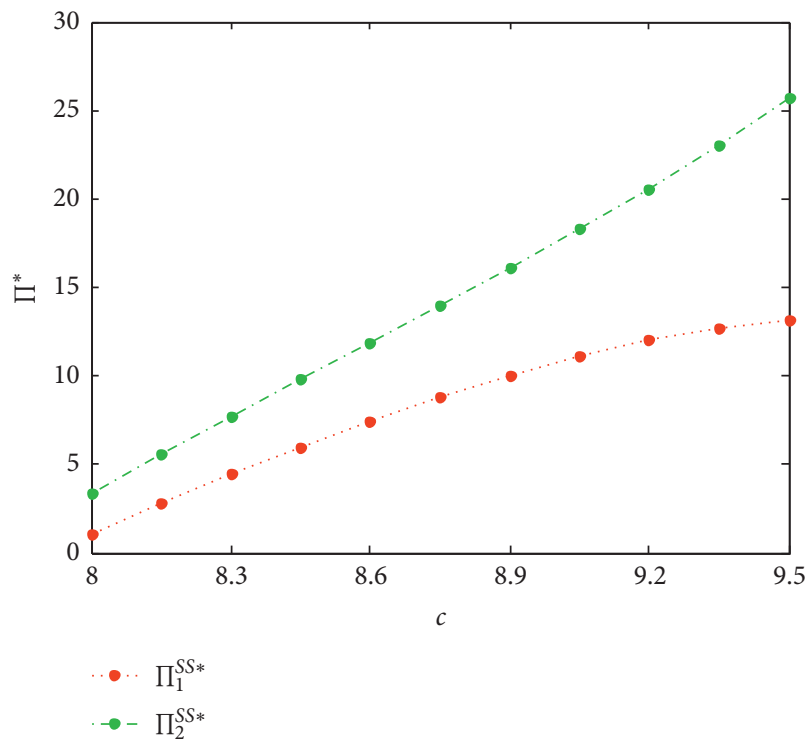

Figure 6: Impact of $c$ on $\Pi^{*}$ under Case SS.

between $\left[0, t_{1 e}\right]$ choose the short-term rental service of platform 1, and the consumers between $\left[t_{2 e}, 1\right]$ choose the long-term rental service of platform 2 , where $t_{1 e}<t_{2 e}$. The profit functions of platform 1 and platform 2 are as follows: $\Pi_{1 e}^{\mathrm{SL}}=r_{1} p_{1 e}^{\mathrm{SL}} t_{1 e} N-c\left(x_{1 e}^{\mathrm{SL}}\right)^{2} \quad$ and $\quad \Pi_{2 e}^{\mathrm{SL}}=r_{2} p_{2 e}^{\mathrm{SL}}\left(1-t_{2 e}\right)-$ $c\left(x_{2 e}^{\mathrm{SL}}\right)^{2}$. According to the first-order conditions of platform 1 's profit function on price and service level, we can find that the price and service level of platform 1 are $p_{1 e}^{\mathrm{SL}}=\left(2 c \lambda v_{S 1} /\left(4 c \lambda-\alpha^{2} r_{1} N\right)\right)$ and $x_{1 e}^{\mathrm{SL}^{*}}=\left(\alpha r_{1} v_{S 1} N /(4 c \lambda-\right.$ $\left.\left.\alpha^{2} r_{1} N\right)\right)$. Therefore, the demand and profit of platform 1 are $D_{1 e}^{S L^{*}}=\left(2 c v_{S 1} /\left(4 c \lambda-\alpha^{2} r_{1} N\right)\right)$ and $\Pi_{1 e}^{S L^{*}}=\left(c r_{1} v_{S 1}^{2} N /(4 c \lambda\right.$ $\left.\left.-\alpha^{2} r_{1} N\right)\right)$. Similarly, the price and service level of platform 2 are $p_{2 e}^{\mathrm{SL}^{*}}=\left(2 \beta c \lambda v_{L 2} /\left(4 \beta c \lambda-\alpha^{2} r_{2} N\right)\right)$ and $x_{2 e}^{\mathrm{SL}}=\left(\alpha r_{2} v_{L 2} /\right.$ $\left.\left(4 \beta c \lambda-\alpha^{2} r_{2} N\right)\right)$, respectively. Furthermore, the demand and profit of platform 2 are $D_{2 e}^{\mathrm{SL}^{*}}=\left(2 c v_{L 2} /\right.$ $\left.\left(4 \beta c \lambda-\alpha^{2} r_{2} N\right) N\right)$ and $\Pi_{2 e}^{S L^{*}}=\left(c r_{2} v_{L 2}^{2} /\left(4 \beta c \lambda-\alpha^{2} r_{2} N\right) N\right)$, respectively.

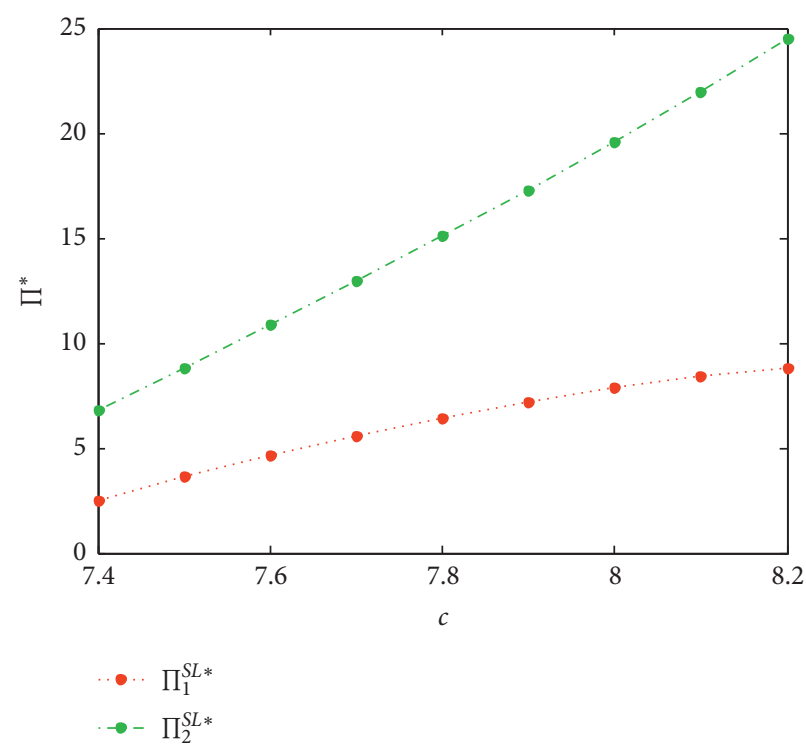

Figure 7: Impact of $c$ on $\Pi^{*}$ under Case SL.

We need to ensure that $0<t_{1 e}<t_{2 e}<1$, so that both platforms can act as local monopolies. To ensure $t_{1 e}<t_{2 e}$, $2 c\left[v_{S 1}\left(4 \beta c \lambda-\alpha^{2} r_{2} N\right) N+v_{L 2}\left(4 c \lambda-\alpha^{2} r_{1} N\right)\right]<\left(4 c \lambda-\alpha^{2} r_{1}\right.$ N) $\left(4 \beta c \lambda-\alpha^{2} r_{2} N\right) N$ must be met. If this condition is not met, the two platforms will directly complete with marginal consumers.

6.3. Case LS: Both Platforms Are Local Monopolies, Platform 1 Chooses the Long-Term Rental Strategy, and Platform 2 Chooses the Short-Term Rental Strategy. When platform 1 chooses the long-term rental strategy and platform 2 chooses short-term rental strategy, the marginal consumers who choose the service of platform 1 are at $t_{1 e}=\left(\left(\alpha x_{1 e}^{\mathrm{LS}} N-p_{1 e}^{\mathrm{LS}}+v_{L 1}\right) / \beta \lambda N\right)$, and the marginal consumers who choose the service of platform 2 are at $t_{2 e}=\left(\left(\lambda+p_{2 e}^{\mathrm{LS}}-\alpha x_{2 e}^{\mathrm{LS}}-v_{L 2}\right) / \lambda\right)$. The consumers between 


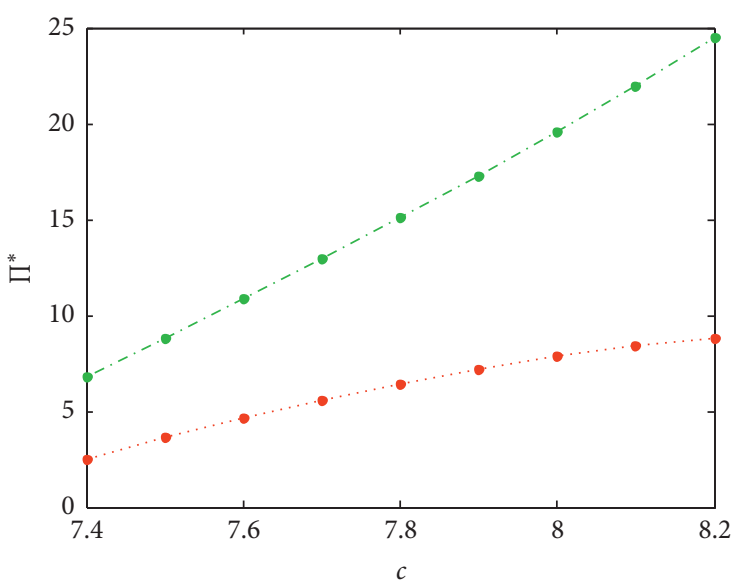

-. $\Pi_{1}^{S L *}$

$\rightarrow \Pi_{2}^{S L *}$

Figure 8: Impact of $c$ on $\Pi^{*}$ under Case LS.

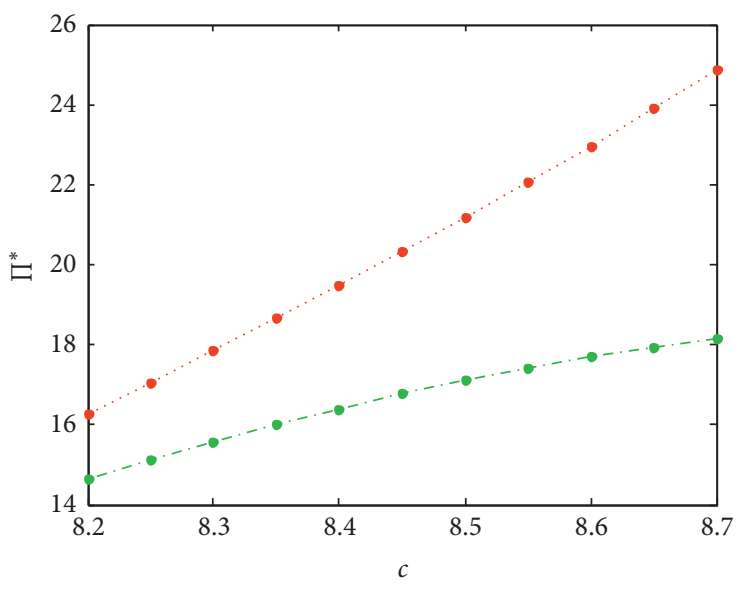

-.. $\Pi_{1}^{L S *}$

Figure 9: Impact of $c$ on $\Pi^{*}$ under Case LL.

$\left[0, t_{1 e}\right]$ choose the long-term rental service of platform 1 , and the consumers between $\left[t_{2 e}, 1\right]$ choose the short-term rental service of platform 2, where $t_{1 e}<t_{2 e}$. The profit functions of platform 1 and platform 2 are as follows: $\Pi_{1 e}^{\mathrm{LS}}=r_{1} p_{1 e}^{\mathrm{LS}} t_{1 e}-$ $c\left(x_{1 e}^{\mathrm{LS}}\right)^{2}$ and $\Pi_{2 e}^{\mathrm{LS}}=r_{2} p_{2 e}^{\mathrm{LS}}\left(1-t_{2 e}\right) N-c\left(x_{2 e}^{\mathrm{LS}}\right)^{2}$. According to the first-order conditions of platform 1's profit function on price and service level, we can find that the price and service level of platform 1 are $p_{1 e}^{L S^{*}}=\left(2 \beta c \lambda v_{S 1} /\left(4 \beta c \lambda-\alpha^{2} r_{1} N\right)\right)$ and $x_{1 e}^{\mathrm{LS}^{*}}=\left(\alpha r_{1} v_{L 1} /\left(4 \beta c \lambda-\alpha^{2} r_{1} N\right)\right)$. Therefore, the demand and profit of platform 1 are $D_{1 e}^{\mathrm{LS}}=\left(2 c v_{L 1} /\left(4 \beta c \lambda-\alpha^{2} r_{1} N\right) N\right)$ and $\Pi_{1 e}^{L S^{*}}=\left(c r_{1} v_{L 1}^{2} /\left(4 \beta c \lambda-\alpha^{2} r_{1} N\right) N\right)$. Similarly, the price and service level of platform 2 are $p_{2 e}^{\mathrm{LS}}=\left(2 c \lambda v_{S 2} /(4 c \lambda-\right.$ $\left.\left.\alpha^{2} r_{2} N\right)\right)$ and $x_{2 e}^{\mathrm{LS}}=\left(\alpha r_{2} v_{S 2} N /\left(4 c \lambda-\alpha^{2} r_{2} N\right)\right)$, respectively. Furthermore, the demand and profit of platform 2 are $D_{2 e}^{\mathrm{LS}^{*}}=\left(2 c v_{S 2} /\left(4 c \lambda-\alpha^{2} r_{2} N\right)\right)$ and $\Pi_{2 e}^{\mathrm{LS}}=\left(c r_{2} v_{S 2}^{2} N /(4 c \lambda-\right.$ $\left.\alpha^{2} r_{2} N\right)$ ), respectively.

We need to ensure that $0<t_{1 e}<t_{2 e}<1$, so that both platforms can act as local monopolies. To ensure $t_{1 e}<t_{2 e}$,
$2 c\left[v_{L 1}\left(4 c \lambda-\alpha^{2} r_{2} N\right)+v_{S 2}\left(4 \beta c \lambda-\alpha^{2} r_{1} N\right) N\right]<\left(4 c \lambda-\alpha^{2} r_{2}\right.$ N) $\left(4 \beta c \lambda-\alpha^{2} r_{1} N\right) N$ must be met. If this condition is not met, the two platforms will directly complete with marginal consumers.

6.4. Case LL: Both Platforms Are Local Monopolies and Both Platforms Choose Long-Term Rental Strategy. When platform 1 and platform 2 choose the long-term rental strategy, the marginal consumers who choose the service of platform 1 are at $t_{1 e}=\left(\left(\alpha x_{1 e}^{\mathrm{LL}} N-p_{1 e}^{\mathrm{LL}}+v_{L 1}\right) / \beta \lambda N\right)$, and the marginal consumers who choose the service of platform 2 are at $t_{2 e}=\left(\left(\beta \lambda N-\alpha x_{2 e}^{\mathrm{LL}} N+p_{2 e}^{\mathrm{LL}}-v_{L 2}\right) / \beta \lambda N\right)$. The consumers between $\left[0, t_{1 e}\right]$ choose the long-term rental service of platform 1 , and the consumers between $\left[t_{2 e}, 1\right]$ choose the long-term rental service of platform 2 , where $t_{1 e}<t_{2 e}$. The profit functions of platform 1 and platform 2 are as follows: $\Pi_{1 e}^{\mathrm{LL}}=r_{1} p_{1 e}^{\mathrm{LL}} t_{1 e}-c\left(x_{1 e}^{\mathrm{LL}}\right)^{2} \quad$ and $\Pi_{2 e}^{\mathrm{LL}}=r_{2} p_{2 e}^{\mathrm{LL}}\left(1-t_{2 e}\right)-c$ $\left(x_{2 e}^{\mathrm{LL}}\right)^{2}$. According to the first-order conditions of platform 1 's profit function on price and service level, we can find that the price and service level of platform 1 are $p_{1 e}^{L^{*}}=(2 \beta c$ $\left.\lambda v_{L 1} /\left(4 \beta c \lambda-\alpha^{2} r_{1} N\right)\right)$ and $x_{1 e}^{\mathrm{LL}}=\left(\alpha r_{1} v_{L 1} /\left(4 \beta c \lambda-\alpha^{2} r_{1} N\right)\right)$. Therefore, the demand and profit of platform 1 are $D_{1 e}^{L L^{*}}=$ $\left(2 c v_{L 1} /\left(4 \beta c \lambda-\alpha^{2} r_{1} N\right) N\right)$ and $\Pi_{1 e}^{\mathrm{LL}^{*}}=\left(c r_{1} v_{L 1}^{2} /(4 \beta c \lambda-\right.$ $\left.\left.\alpha^{2} r_{1} N\right) N\right)$. Similarly, the price and service level of platform 2 are $p_{2 e}^{\mathrm{LL}^{*}}=\left(2 \beta c \lambda v_{L 2} /\left(4 \beta c \lambda-\alpha^{2} r_{2} N\right)\right)$ and $x_{2 e}^{\mathrm{LL}}=\left(\alpha r_{2} v_{L 2} /(4\right.$ $\left.\beta c \lambda-\alpha^{2} r_{2} N\right)$ ), respectively. Furthermore, the demand and profit of platform 2 are $D_{2 e}^{\mathrm{LL}}=\left(2 c v_{L 2} /\left(4 \beta c \lambda-\alpha^{2} r_{2} N\right) N\right)$ and $\Pi_{2 e}^{\mathrm{LL}}=\left(c r_{2} v_{L 2}^{2} /\left(4 \beta c \lambda-\alpha^{2} r_{2} N\right) N\right)$, respectively.

We need to ensure that $0<t_{1 e}<t_{2 e}<1$, so that both platforms can act as local monopolies. To ensure $t_{1 e}<t_{2 e}$, $2 c\left[v_{L 1}\left(4 \beta c \lambda-\alpha^{2} r_{2} N\right)+v_{L 2} \quad\left(4 \beta c \lambda-\alpha^{2} r_{1} N\right)\right]<\left(4 \beta c \lambda-\alpha^{2}\right.$ $\left.r_{1} N\right)\left(4 \beta c \lambda-\alpha^{2} r_{2} N\right) N$ must be met. If this condition is not met, the two platforms will directly complete with marginal consumers.

\section{Conclusion}

In this paper, we examine a duopoly housing sharing platform market, in which two platforms provide rental services to consumers. Each platform can choose the shortterm rental strategy or the long-term rental strategy. When two platforms make decisions, there are four cases (i.e., Case SS, Case SL, Case LS, and Case LL). The two platforms determine the optimal price and service level to maximize their own profits, based on the competitors' observed decisions. In this paper, the equilibrium price and profit are given by using game theory, and the market equilibrium is analyzed to determine the applicable conditions of each strategy.

The results show that whether the platform adopts the short-term rental strategy or the long-term rental strategy largely depends on the service quality of competitors. Specifically, when the difference of service value is small, it is better for the two platforms to adopt the short-term rental strategy, and when the difference of services value is large, the two platforms intend to adopt the long-term rental strategy. We also find that there may be two equilibria under certain conditions, and we also give the conditions of 
dominant equilibrium. In addition, we also find that the commission rate of the two platforms will affect the profits of the platforms, and when the service quality is fixed, the platform can benefit from the decreasing service cost. Finally, we analyze the uncovered market and get the equilibrium profits of the two platforms.

Our results can provide some important management implications for the strategic choice of home-sharing platforms in the competitive home-sharing market. First, when a platform plans to choose the short-term rental strategy or the long-term rental strategy, it needs to compare the service quality with its competitors. Each platform should build its own user base to improve profits. Second, when the platform and the competitor have the same or almost the same level of service quality, the platform will choose the strategy according to the intrinsic value of the competitor's service. Finally, the commission rate or service cost will also affect the profits of the platforms. Therefore, these management implications provide a reference for home-sharing platforms in different cases.

Future work can also be carried out in two aspects. First, we focus on all listed homes on the commercial operator control platform in this paper. In some home-sharing platforms, commercial operators and individual hosts also coexist. Second, the home-sharing platform holds a large number of data, and the influence of data-driven on the operational decision of the home-sharing platform can be considered in the future.

\section{Appendix}

Proof. of Lemma 1.

By $U_{S 1}=U_{S 2}$, we can obtain $t_{0}=\left(\left(\lambda-p_{1}^{S S}+p_{2}^{S S}+v_{S 1}-v_{S 2}+\alpha x_{1}^{S S}-\alpha x_{2}^{S S}\right) / 2 \lambda\right)$. Furthermore, $D_{\mathrm{SS}}^{1}=t_{0}$ and $D_{\mathrm{SS}}^{2}=1-t_{0}$. Substituting $D_{\mathrm{SS}}^{1}$ and $D_{\mathrm{SS}}^{2}$ into equations (5) and (6), respectively, we can get

$$
\begin{aligned}
& \Pi_{1}^{\mathrm{SS}}=r_{1} p_{1}^{\mathrm{SS}} D_{1}^{\mathrm{SS}} N-c\left(x_{1}^{\mathrm{SS}}\right)^{2}, \\
& \Pi_{2}^{\mathrm{SS}}=r_{2} p_{2}^{\mathrm{SS}} D_{2}^{\mathrm{SS}} N-c\left(x_{2}^{\mathrm{SS}}\right)^{2} .
\end{aligned}
$$

Let $\left(\partial \Pi_{1}^{S S} / \partial p_{1}^{S S}\right)=0,\left(\partial \Pi_{1}^{S S} / \partial x_{1}^{S S}\right)=0,\left(\partial \Pi_{2}^{S S} / \partial p_{2}^{S S}\right)=0$, and $\left(\partial \Pi_{2}^{S S} / \partial x_{2}^{S S}\right)=0$, and solve them simultaneously; we can get $p_{1}^{S S^{*}}=\left(2 \lambda\left(6 c \lambda-\alpha^{2} r_{2} N+2 c v_{S 1}-2 c v_{S 2}\right) /\left(12 c \lambda-\alpha^{2} r_{1} N\right.\right.$ $\left.\left.-\alpha^{2} r_{2} N\right)\right), \quad x_{1}^{\mathrm{SS} *}=\left(\alpha r_{1} N\left(6 c \lambda-\alpha^{2} r_{2} N+2 c v_{S 1}-2 c v_{S 2}\right) /\right.$ $\left.2 c\left(12 c \lambda-\alpha^{2} r_{1} N-\alpha^{2} r_{2} N\right)\right), p_{2}^{S S^{*}}=\left(2 \lambda\left(6 c \lambda-\alpha^{2} r_{1} N-2 c v_{S 1}\right.\right.$ $\left.\left.+2 c v_{S 2}\right) /\left(12 c \lambda-\alpha^{2} r_{1} N-\alpha^{2} r_{2} N\right)\right)$, and $x_{2}^{\mathrm{SS}^{*}}=\left(\alpha r_{2} N(6 c \lambda-\right.$ $\left.\left.\alpha^{2} r_{1} N-2 c v_{S 1}+2 c v_{S 2}\right) / 2 c\left(12 c \lambda-\alpha^{2} r_{1} N-\alpha^{2} r_{2} N\right)\right)$.

Through the analysis, we know that they are the optimal solutions of models (5) and (6) under the conditions of $8 c \lambda$ $\alpha^{2} r_{1} N>0$ and $8 c \lambda-\alpha^{2} r_{2} N>0$.

Therefore, Lemma 1 holds.

The proof process of the remaining lemmas is similar to that of Lemma 1 , which is not discussed here.

Proof. of Proposition 1. (i) When $\Pi_{1}^{\mathrm{SS}^{*}}>\Pi_{1}^{\mathrm{LS}^{*}}, \Pi_{2}^{\mathrm{SS}^{*}}>\Pi_{2}^{\mathrm{SL}^{*}}$, Case SS is an equilibrium.

(ii) When $\Pi_{1}^{S L^{*}}>\Pi_{1}^{L^{*}}, \Pi_{2}^{\mathrm{SL}^{*}}>\Pi_{2}^{S S^{*}}$, Case SL is an equilibrium.

(iii) When $\Pi_{1}^{\mathrm{LS}^{*}}>\Pi_{1}^{\mathrm{SS}}, \Pi_{2}^{\mathrm{LS}}>\Pi_{2}^{\mathrm{LL}}$, Case LS is an equilibrium.

(iv) When $\Pi_{1}^{\mathrm{LL}^{*}}>\Pi_{1}^{\mathrm{SL}}, \Pi_{2}^{\mathrm{LL}}>\Pi_{2}^{\mathrm{LS}}{ }^{*}$, Case $\mathrm{LL}$ is an equilibrium.

We can solve (i)-(iv) separately and get the conditions of each equilibrium. The solution process will not be discussed.

Therefore, Proposition 1 holds.

The proof process of the remaining propositions is similar to that of Proposition 1, which is not discussed here.

\section{Data Availability}

The data used to support the findings of the study are included within the article.

\section{Conflicts of Interest}

The authors declare that they have no conflicts of interest.

\section{Authors' Contributions}

Yingna Li and Pengfei Ma designed the study, performed the research, and wrote the paper.

\section{Acknowledgments}

It is gratefully acknowledged that this study was supported by the Department of Management Engineering, Inner Mongolia Vocational and Technical College of Communications, Chifeng, China.

\section{References}

[1] T. A. Taylor, "On-demand service platforms," Manufacturing and Service Operations Management, vol. 20, no. 4, pp. 704720, 2018.

[2] H. Li and K. Srinivasan, "Competitive dynamics in the sharing economy: an analysis in the context of Airbnb and hotels," Marketing Science, vol. 38, no. 3, pp. 365-391, 2019.

[3] T. Ikkala and A. Lampinen, "Monetizing network hospitality: hospitality and sociability in the context of Airbnb," in Proceedings of the 18th ACM Conference on Computer Supported Cooperative Work and Social Computing, pp. 10331044, Vancouver, BC, Canada, March 2015.

[4] G. Quattrone, D. Proserpio, D. Quercia, and L. Capra, "Who benefits from the "sharing" economy of Airbnb?" in Proceedings of the 25th International Conference on World Wide Web, pp. 1385-1394, Montréal, Québec, Canada, April 2016.

[5] G. Bensinger, "Airbnb valued at $\$ 31$ billion after new funding round," The Wall Street Journal, 2017, https://www.wsj.com/ articles/airbnb-valued-at-31-billion-after-new-funding-round1489086240.

[6] Airbnb, https://press.airbnb.com/about-us/, 2020.

[7] H. Zhu, M. Gumus, and S. Ray, "Short-term housing rentals and corporatization of platform pricing,", 2019. 
[8] Curbed, "Airbnb business: as professionals find success on the platform, is there still room for sharers?," 2018, https://www. curbed.com/2018/2/21/17032100/airbnb-business-profit-hotelproperty-management.

[9] CityLab, What Airbnb Did to New York City, CityLab, New York, NY, USA, 2018, https://www.citylab.com/equity/2018/ 03/what-airbnb-did-to-new-york-city/552749/.

[10] D. Wang and J. L. Nicolau, "Price determinants of sharing economy based accommodation rental: a study of listings from 33 cities on Airbnb.com," International Journal of Hospitality Management, vol. 62, pp. 120-131, 2017.

[11] G. P. Cachon, K. M. Daniels, and R. Lobel, "The role of surge pricing on a service platform with self-scheduling capacity," Manufacturing and Service Operations Management, vol. 19, no. 3, pp. 368-384, 2017.

[12] W. Liu, X. Yan, W. Wei, and D. Xie, "Pricing decisions for service platform with provider's threshold participating quantity, value-added service and matching ability," Transportation Research Part E: Logistics and Transportation Review, vol. 122, pp. 410-432, 2019.

[13] N. Irtija, F. Sangoleye, and E. E. Tsiropoulou, "Contracttheoretic demand response management in smart grid systems," IEEE Access, vol. 8, pp. 184976-184987, 2020.

[14] H. Jiang, W. Liu, G. Jiang et al., "Fly-Navi: a novel indoor navigation system with on-the-fly map generation," IEEE Transactions on Mobile Computing, vol. 20, no. 9, pp. 28202834, 2021.

[15] D. Liu, Z. Cao, M. Hou, H. Rong, and H. Jiang, "Pushing the limits of transmission concurrency for low power wireless networks," ACM Transactions on Sensor Networks, vol. 16, no. 4, pp. 1-29, 2020.

[16] Y.-W. Zhou, X. Lin, Y. Zhong, and W. Xie, "Contract selection for a multi-service sharing platform with self-scheduling capacity," Omega, vol. 86, pp. 198-217, 2019.

[17] C. Gibbs, D. Guttentag, U. Gretzel, L. Yao, and J. Morton, "Use of dynamic pricing strategies by Airbnb hosts," International Journal of Contemporary Hospitality Management, vol. 30, no. 1, pp. 2-20, 2018.

[18] E. Gal-Or, "Peer-to-peer sharing in the lodging market: evaluating implications for social welfare and profitability," Journal of Economics and Management Strategy, vol. 27, no. 4, pp. 686-704, 2018.

[19] E. Gal-Or, "How does competition among lodging sharing platforms affect welfare and profits?" Journal of Economics and Management Strategy, vol. 30, no. 1, pp. 45-62, 2021.

[20] J. Li, A. Moreno, and D. Zhang, Pros vs. Joes: Agent Pricing Behavior in the Sharing Economy, no. 1298, Ross School of Business Paper, 2016.

[21] J. Li, F. Zeng, Z. Xiao et al., "Drive2friends: inferring social relationships from individual vehicle mobility data," IEEE Internet of Things Journal, vol. 7, no. 6, pp. 5116-5127, 2020.

[22] H. Jiang, W. Liu, G. Jiang et al., "Fly-Navi: a novel indoor navigation system with on-the-fly map generation," IEEE Transactions on Mobile Computing, vol. 20, no. 9, pp. 28202834, 2021.

[23] Z. Xiao, X. Dai, H. Jiang et al., "Vehicular task offloading via heat-aware MEC cooperation using game-theoretic method," IEEE Internet of Things Journal, vol. 7, no. 3, pp. 2038-2052, 2019.
[24] I. Hong and C. Yoo, "Analyzing spatial variance of Airbnb pricing determinants using multiscale GWR approach," Sustainability, vol. 12, no. 11, p. 4710, 2020.

[25] G. Zervas, D. Proserpio, and J. W. Byers, "The rise of the sharing economy: estimating the impact of Airbnb on the hotel industry," Journal of Marketing Research, vol. 54, no. 5, pp. 687-705, 2017.

[26] K. Barron, E. Kung, and D. Proserpio, The Sharing Economy and Housing Affordability: Evidence from Airbnb, 2018. 